\title{
CORRECTION
}

\section{Correction: Sex-specific relationships between early nutrition and neurodevelopment in preterm infants}

\author{
Anna C. Tottman, Frank H. Bloomfield, Barbara E. Cormack, Jane E. Harding, Janice Taylor and Jane M. Alsweiler
}

Pediatric Research (2022) 91:1627; https://doi.org/10.1038/s41390-021-01582-8

Correction to: Pediatric Research https://doi.org/10.1038/s41390019-0695-y, published online 29 November 2019

The authors would like to correct a sentence in the third paragraph of the "Discussion" section to read as follows:

In our study, fat intake in the first postnatal week was strongly associated with survival without neurodevelopmental impairment in girls but not boys. The reason for the association between fat intake and outcome in girls, and the absence of this association in boys, is not clear, but could be due to differences in the requirements for, and metabolism of, fatty acids in early life. At 1 month of age, term-born girls have a higher percentage body fat and less fat-free mass than boys. ${ }^{32}$ Healthy infant girls have higher serum concentrations of triglycerides, cholesterol and low density lipoproteins than boys, ${ }^{33}$ and also different lipid compositions of the vernix (which is dissolved into the amniotic fluid by surfactant proteins then swallowed by the fetus ${ }^{34,35}$ ), with girls tending towards longer-chain hydrocarbon components. ${ }^{36}$ Fish oil supplementation to lactating mothers of term infants was associated with increased diastolic blood pressure and delayed puberty at 13 years only in boys. ${ }^{37}$ However, a long-term study of docosahexaenoic acid supplementation to preterm neonates showed worsened executive function only in girls. ${ }^{38}$ Together, these findings suggest sexual dimorphism in lipid requirement and metabolism, and hence potentially nutritional fat requirements in early life.

Publisher's note Springer Nature remains neutral with regard to jurisdictional claims in published maps and institutional affiliations. 